\title{
Adult growth hormone deficiency guidelines: more difficult than it seems to incorporate into clinical practice universally
}

\section{Kevin C J Yuen (1)}

Departments of Neuroendocrinology and Neurosurgery, Barrow Pituitary Center, Barrow Neurological Institute, University of Arizona College of Medicine and Creighton School of Medicine, Phoenix, Arizona, USA
Correspondence should be addressed to $\mathrm{K}$ C J Yuen Email

Kevin.Yuen@DignityHealth. org

\begin{abstract}
Adult growth hormone deficiency (GHD) is a syndrome characterized by adverse phenotypic, metabolic, and qualityof-life features. Over the past 2 decades, there is accumulating evidence demonstrating improvement of most of these parameters when GH is optimally replaced. Appropriate selection of patients at risk of GHD is crucial when considering and performing testing to establish the diagnosis. While generally safe, GH replacement requires careful dose initiation and monitoring to assure effectiveness and tolerance in treated patients. Several consensus clinical practice guidelines recommend evaluation of adults presenting with hypothalamic-pituitary disorders for GHD. However, the clinical practice of managing such patients varies among countries largely due to lack of recognition of the condition, lack of GH availability, and lack of reimbursement of the drug, as demonstrated from a large online survey prepared by the European Society of Endocrinology involving 2148 patients from Europe and Australia. These data reinforce the notion of the large variability of disease recognition, clinical practice and education of adult GHD amongst healthcare professionals, and the lack of availability and reimbursement of the drug contributing to the under-utilization of GH replacement therapy in several countries. This commentary article highlights the fact that despite the publication of several guideline recommendations and positive long-term safety and efficacy data of GH replacement, there is still a need for increased education to enhance the awareness in the general population and improve the knowledge of healthcare professionals and administrators of adult GHD as a disease state to allow for early identification and treatment optimization.
\end{abstract}

Adult growth hormone deficiency (GHD) is a clinical entity characterized by numerous adverse phenotypic, metabolic and quality-of-life features and has been thought to be associated with premature mortality (1). However, as the presenting symptoms and signs of this clinical entity are typically non-specific, clinicians are recommended by consensus clinical practice guidelines to perform diagnostic testing only in adults with a reasonable probability of GHD (e.g. patients with a history of hypothalamicpituitary disorders and those that had undergone surgery and/or cranial irradiation to this region in the brain), and with the intention to offer treatment when the diagnosis is established $(2,3,4)$. Since recombinant human GH was approved over 25 years ago in Europe and the United States, there are now extensive efficacy and safety data supporting its use in adults with GHD $(5,6)$.

Clinical practice guidelines have become increasingly prominent in clinical medicine over the last 4 decades, and represent one of the most important tools for improving clinical decision-making, patient understanding and potentially patient outcomes. Several professional societies (e.g. the European Society of Endocrinology, Growth Hormone Research Society, The Endocrine Society and the American Association of Clinical Endocrinology) have

Published by Bioscientifica Ltd. 
published their clinical practice guidelines that provide comprehensive recommendations regarding the appropriate screening, identification, and treatment strategies for a range of individuals with various causes of adult $\operatorname{GHD}(2,3,4)$. It is, however, noteworthy that these guidelines are developed from the body of established literature (evidencebased) complemented by expert opinion (consensus-based). The key distinction between these two approaches is that when there is high quality evidence, recommendations are made based on the evidence that speaks for itself, whereas if the evidence is insufficient or of low quality, recommendations are mainly consensus-based. Whether the available evidence is considered as being of high or low quality require careful consideration and consensus from the expert panel regarding the interpretation of the evidence and the trade-off between benefit vs harm or burden of the recommended testing and drug intervention. While educational and helpful to the clinician, many recommendations from such clinical practice guidelines may not be applicable universally. In fact, in some countries, adult GHD has been perceived as low priority, hence explaining the lack of availability of reimbursement for the drug. Problems also arise due to the lack of acceptance of the clinical entity of adult GHD per se, the lack of perception of the benefits of GH replacement therapy by some healthcare professionals and administrators, high cost of therapy, the lack of availability and variable performance of diagnostic tests in terms of accuracy, reproducibility and local resources, the heterogeneity of current GH and IGF-I assays, the lack of agreement of the optimal target IGF-I level to be achieved during GH therapy, subjectivity of the effects of GH on the improvement of quality of life and the lack of data of the effects of GH on more robust outcomes, for example, mortality and fracture rates.

The recent study by Martel-Duguech et al. (7) utilizing the data derived from endocrinologists who voluntarily completed an electronic questionnaire is timely and interesting in addressing the question of how current clinical practices of assessing and treating adult GHD compare with existent clinical practice guidelines used as a benchmark, and how the condition is perceived in the endocrine community in different countries. Under the auspices of the European Society of Endocrinology, this initiative brings together a large wealth of 'real-world' clinical practice data of experienced endocrinologists from 17 European countries and Australia who treat ten or more adults with GHD in their clinical practice. The striking feature of this audit is the demonstration of the heterogeneity of healthcare systems across various countries, the under-appreciation of the benefits and perhaps, also the likely reason for under- utilization of GH replacement therapy in some countries, and the lack of availability, indication for testing (especially those with cranial irradiation and traumatic brain injury) and reimbursement of the drug in some countries, including one country where reimbursement of treatment remains unapproved. These findings highlight a sobering fact that despite the publications of several guideline recommendations and overwhelming evidence indicating the beneficial effects and long-term safety of GH therapy in adults with GHD. Additionally, this audit also reinforces an unmet need that prompts calls to increase the disease awareness of adult GHD in trainee endocrinologist and nonendocrine healthcare professionals through education in order to embrace the condition based on available evidence to allow for early identification and optimal treatment of this condition.

The majority of patients in this study were adultonset patients, and there was no treatment initiation delay after the diagnosis of adult GHD was established. Appropriate types of $\mathrm{GH}$-stimulation testing were used that is largely dependent on test availability and local resources in different countries. Most centres used the lower GH diagnostic cut-off for the insulin tolerance test of $<3 \mu \mathrm{g} / \mathrm{L}$ rather than $<5 \mu \mathrm{g} / \mathrm{L}$ to decrease the likelihood of false positive tests and to allow only those with severe GHD to be considered for GH replacement. This study also revealed several interesting clinical practices that differ to the recommendations proposed by the existent clinical practice guidelines. Although there are data to suggest the BMI effects in impacting the accuracy of the GHRH+arginine test, some countries like Italy and Denmark do not take BMI into consideration when interpreting the results of this test. Conversely, countries like Romania, Portugal, Bulgaria and Serbia would test patients for adult GHD, but many patients were not treated due to administrative issues (e.g. lack of reimbursement and restrictions to provide drug in hospital pharmacies), which begs the question of why test the patient if no treatment is going to be offered after the diagnosis is established. In Portugal and Australia, the practice of weight-based GH initial dosing remains despite being discouraged by existent guidelines to use fixed low-dose regimens due to concerns of possible induction of side-effects (e.g. hyperglycaemia) with higher GH doses in higher BMI individuals $(2,3,4)$. As for transition paediatric patients to adult services, the reasons for treatment cessation appear to be similar to previous published studies (8). This audit also revealed that monitoring for changes in quality of life is not universally assessed as only $45 \%$ of patients were evaluated, suggesting that this parameter is not generally 
perceived to be a major symptom of GH effect, although in clinical practice, improvement in quality of life is an important measure of treatment efficacy and patient motivation to remain adherent to long-term GH therapy $(2,3,4)$. Additionally, the lack of reimbursement appears to be a major prohibitive factor in several countries to its wider use. Because the respondents of this online survey are experienced clinicians who are in acceptance of the use of GH replacement therapy in adults with GHD, it would be of interest to get a sense of the views of the healthcare professionals and administrators who do not recognize the clinical entity of adult GHD. The questions that could be explored include whether these individuals would be amenable to being educated in greater depth about the disease and alerted to the benefits and safety of long-term GH therapy (particularly differentiating between treating bona fide adult GHD vs the utilization of GH therapy for sporting enhancement or for anti-aging purposes that is strongly discouraged), and after being educated, whether they would be amenable to consider GH replacement therapy in adults with GHD.

For the time being, concerted efforts are needed to increase disease awareness by educating the general population, medical students, endocrine trainees, nonendocrine healthcare professionals and administrators to embrace the condition and allow for early detection and treatment of adult GHD based on recommendations proposed by clinical practice guidelines. An important question is whether the onus of these educational efforts should fall on the shoulders of endocrinologists? The short answer is 'yes', especially from endocrinologists with extensive experience in treating this condition. That said, professional societies (e.g. the European Society of Endocrinology, The Endocrine Society and the American Association of Clinical Endocrinology) could also play a greater role in promoting disease awareness to the general population and other non-endocrine healthcare professional societies of the benefits and safety of GH replacement therapy in adults with GHD. Finally, with macimorelin now approved as a diagnostic test in Europe and the United States and the likelihood of some long-acting GH preparations gaining regulatory approval in the near future, it would also be of interest to assess the reception of these new compounds amongst endocrine healthcare professionals and administrators as to whether more GH-stimulation testing would be performed and long-acting GH preparations utilized in treating adult GHD.

\section{Declaration of interest}

The author declares having served as an occasional consultant to Novo Nordisk, Sandoz and Ascendis.

\section{Funding}

This commentary article did not receive any specific grant from any funding agency in the public, commercial or not-for-profit sector.

\section{References}

1 Simpson H, Savine R, Sonksen P, Bengtsson BA, Carlsson L, Christiansen JS, Clemmons D, Cohen P, Hintz R, Ho K et al. Growth hormone replacement therapy for adults: into the new millennium. Growth Hormone and IGF Research 200212 121-133. (doi: 10.1054/ ghir.2001.0263)

2 Ho KK \& GH. Consensus guidelines for the diagnosis and treatment of adults with GH deficiency II: a statement of the GH Research Society in Association with the European Society for Pediatric Endocrinology, Lawson Wilkins Society, European Society of Endocrinology, Japan Endocrine Society, and Endocrine Society of Australia. European Journal of Endocrinology 2007157 695-700. (https://doi.org/10.1530/EJE-07-0631)

3 Molitch ME, Clemmons DR, Malozowski S, Merriam GR \& Vance ML. Evaluation and treatment of adult growth hormone deficiency: an Endocrine Society clinical practice guideline. Journal of Clinical Endocrinology and Metabolism 201196 1587-1609. (https://doi. org/10.1210/jc.2011-0179)

4 Yuen KCJ, Biller BMK, Radovick S, Carmichael JD, Jasim S, Pantalone KM \& Hoffman AR. American Association of Clinical Endocrinologists and American College of Endocrinology Guidelines for management of growth hormone deficiency in adults and patients transitioning from pediatric to adult care. Endocrine Practice 201925 1191-1232. (https://doi.org/10.4158/GL-2019-0405)

5 Elbornsson M, Gotherstrom G, Bosaeus I, Bengtsson BÅ, Johannsson G \& Svensson J. Fifteen years of GH replacement improves body composition and cardiovascular risk factors. European Journal of Endocrinology 2013168 745-753. (https://doi.org/10.1530/ EJE-12-1083)

6 Rochira V, Mossetto G, Jia N, Cannavo S, Beck-Peccoz P, Aimaretti G, Ambrosio MR, Di Somma C, Losa M, Ferone D et al. Analysis of characteristics and outcomes by growth hormone treatment duration in adult patients in the Italian cohort of the Hypopituitary Control and Complications Study (HypoCCS). Journal of Endocrinological Investigation 201841 1259-1266. (https://doi.org/10.1007/s40618-018-0860-x)

7 Martel-Duguech LM, Jorgensen JOL, Korbonits M, Johannsson G, Webb SM, Amadidou F, Mintziori G, Arosio M, Giavoli C, Badiu C et al. ESE audit on management of adult growth hormone deficiency in clinical practice. European Journal of Endocrinology 2020 EJE-201180.R1. (https://doi.org/10.1530/EJE-20-1180)

8 Yuen KCJ, Alter CA, Miller BS, Gannon AW, Tritos NA, Samson SL, Dobri G, Kurtz K, Strobl F \& Kelepouris N. Adult growth hormone deficiency: optimizing transition of care from pediatric to adult services. Growth Hormone and IGF Research 202056 101375. (https:// doi.org/10.1016/j.ghir.2020.101375) 